3. Samuel Eilenberg and Saunders MacLane, On the groups $H(\pi, n)$ I, Ann. of Math. vol. 58 (1953) pp. 55-106.

4. - On the groups $H(\pi, n)$ II, Ann. of Math. vol. 60 (1954) pp. 49-139.

5. - On the groups $H(\pi, n)$ III, Ann. of Math. vol. 60 (1954) pp. 513-557.

6. - Relations between homology and homotopy groups of spaces II, Ann. of Math. vol. 51 (1950) pp. 514-533.

7. J.-P. Serre, Homologie singulière des espaces fibrés. Applications, Ann. of Math. vol. 54 (1951) pp. 425-505.

8. - Groupes d'homotopie et classes de groupes abelians, Ann. of Math. vol. 58 (1953) pp. 258-294.

9. G. Whitehead, On the characteristic cohomology class of a fibre bundle, Bull. Amer. Math. Soc. Abstract 55-5-261.

The University of Chicago and

The University of AbERDEen

\title{
TEST SPACES FOR DIMENSION $n$
}

\section{BYRON H. MCCANDLESS}

In 1935 Hurewicz published his celebrated theorem characteriaing the dimension of separable metric spaces by means of mappings into the $n$-sphere $S^{n}$. In this note we shall be concerned with the "dimension testing" property which $S^{n}$ displays in Hurewicz's theorem. That is, we shall try to determine the properties of a space $Y^{n}$ which enable it to characterize the dimension of separable metric spaces. Let us agree from the outset that only nonempty separable metric spaces will be considered in this problem. To make the above more precise, let $n$ be a non-negative integer and $Y^{n}$ a separable metric space. In case $Y^{n}$ satisfies the statement:

A separable metric space $X$ has dimension $\leqq n$ if, and only if, given a closed subset $C$ of $X$ and mapping $f: C \rightarrow Y^{n}$ there is an extension of $f$ over $X$, we shall call $Y^{n}$ a test space for dimension $n$. Our problem, then, is to characterize these spaces $Y^{n}$.

A special case of the problem seems to be general knowledge, although I have seen no mention of it in the literature. It is known, namely, that an arcwise connected space $Y^{n}$ tests the dimension of locally finite $k$-polytopes $(1 \leqq k \leqq n)$ if and only if $\pi_{i}\left(Y^{n}\right)=0, i<n$,

Presented to the Society, February 25, 1956; received by the editors November 14, 1955 and, in revised form, January 4, 1956.

1 Supported in part by the Research Council of Rutgers University. 
and $\pi_{n}\left(Y^{n}\right) \neq 0$. Here, as usual, $\pi_{i}\left(Y^{n}\right)$ denotes the $i$-dimensional homotopy group of the space $Y^{n}$.

Let $E^{n+1}$ be the solid $(n+1)$-dimensional sphere and $S^{n}$ its boundary. A space $Y$ is said to be locally connected in dimension $n$ at a point $y_{0}$ of $Y$ if, given any $\epsilon>0$, there exists a $\delta>0$ such that every mapping $f: S^{n} \rightarrow N_{\delta}\left(y_{0}\right)$ has an extension $F: E^{n+1} \rightarrow N_{\epsilon}\left(y_{0}\right)$. If $Y$ is locally connected in dimension $n$ at each of its points, we say that $Y$ is locally connected in dimension $n$ ( notation: $n$-LC). In case $Y$ is locally connected in all dimensions $\leqq n$, we write $\mathrm{LC}^{n}$.

In Theorem 2 we shall use the idea of regularly aspheric spaces. According to Hurewicz [2, p. 216], an arcwise connected space $Y$ is aspheric if $\pi_{i}(Y)=0, i=2,3, \cdots$. An aspheric space is regularly aspheric if it is locally connected in all dimensions. In particular [2, p. 216], every compact one-dimensional, connected, locally contractible space is regularly aspheric.

Following Kuratowski [1], we shall use the symbol $X \tau Y$ to mean that if $C$ is a closed subset of $X$ and $f: C \rightarrow Y$ is any mapping, then there is an extension of $f$ over $X$. By $\pi_{0}(Y)=0$, we shall mean that $Y$ is arcwise connected.

We now list four of the five properties of the space $Y^{n}(n \geqq 1)$ which we will be interested in:

$$
\begin{aligned}
& 1^{n} . X \tau Y^{n} \text { implies } \operatorname{dim} X \leqq n . \\
& 2^{n} . \operatorname{dim} X \leqq n \text { implies } X \tau Y^{n} . \\
& 3^{n} . \pi_{i}\left(Y^{n}\right)=0, i=0,1, \cdots, n-1 . \\
& 4^{n} . Y^{n} \text { is } \mathrm{LC}^{n-1} .
\end{aligned}
$$

Clearly, $Y^{n}$ is a test space for dimension $n$ if and only if it has properties $1^{n}$ and $2^{n}$.

REMARK. It is easy to see that $\operatorname{dim} X=0$ implies $X \tau Y$ for any space $Y$. For a theorem of Borsuk [6] shows that a closed subset of a 0 -dimensional space is a retract of the space.

Leмma 1. If $n$ is an integer $\geqq 1$ and $Y^{n}$ has property $2^{n}$, then $\pi_{0}\left(Y^{n}\right)$ $=0$.

The proof is trivial.

THEOREM 1. If $n$ is a positive integer, then a space $Y^{n}$ has property $2^{n}$ if and only if it has properties $3^{n}$ and $4^{n}$.

This is a theorem of Kuratowski [1, pp. 265-266].

REMARK. If $n$ is a positive integer, a space $Y^{n}$ with properties $1^{n}$ and $2^{n}$ has dimension $\geqq n$. For otherwise, property $2^{n}$ implies that 
$Y^{n}$ is an absolute retract (see $[1$, p. 289]) and cannot have the property $1^{n}$.

LemMA 2. Let $n$ be an integer $\geqq 1$. If $Y^{n}$ is $n$-LC and has properties $1^{n}$ and $2^{n}$, then $\pi_{n}\left(Y^{n}\right) \neq 0$.

Proof. If the lemma is false, then $Y^{n}$ has properties $3^{n+1}$ and $4^{n+1}$ by Theorem 1 , and therefore has property $2^{n+1}$. But property $1^{n}$ shows that this is impossible.

THEOREM 2. Let $n$ be an integer $\geqq 1$, and suppose that $Y^{n}$ is compact, $n$-dimensional, and $n$-LC. If $n=1$, we also assume that $Y^{1}$ is 1-simple (i.e., $\pi_{1}\left(Y^{1}\right)$ is abelian). Then if $Y^{n}$ has properties $1^{n}$ and $2^{n}$, the following statements are true: If $n=1$, then $\pi_{1}\left(Y^{1}\right)$ is infinite cyclic, and if $n \geqq 2$ there exists an integer $k_{n}>0$ such that $\pi_{n}\left(Y^{n}\right)$ is the direct sum of $k_{n}$ infinite cyclic groups.

Proof. By Lemma 2, $\pi_{n}\left(Y^{n}\right) \neq 0$.

We first consider the case $n=1$. Since $\operatorname{dim} Y^{1}=1$ and $Y^{1}$ is $\mathrm{LC}^{1}$, it is clear that $Y^{1}$ is locally contractible. Property $2^{1}$ shows that $Y^{1}$ is connected, and thus we conclude that $Y^{1}$ is regularly aspheric by our remark above. Some results of Hurewicz now show that $\pi_{1}\left(Y^{1}\right)$ has no elements of finite order, other than the identity $[2$, p. 216], and that $\pi_{1}\left(Y^{1}\right)$ has just one free generator $[2$, p. 222].

Now suppose $n \geqq 2$. $\pi_{n}\left(Y^{n}\right)$ is, of course, isomorphic to the $n$th homology group of $Y^{n}$ with integral coefficients by Hurewicz's wellknown Isomorphism Theorem. Since $\operatorname{dim} Y^{n}=n$, then, we see that $\pi_{n}\left(Y^{n}\right)$ can have no elements of finite order, other than the identity $\left[2\right.$, p. 119]. On the other hand, $Y^{n}$ is $\mathrm{LC}^{n}$ and therefore $\pi_{n}\left(Y^{n}\right)$ has a finite number, say $k_{n}$, of generators $[2$, p. 222 , footnote]. This completes the proof.

We now list the fifth property of the space $Y^{n}$ which we will consider:

$$
5^{n} \cdot \pi_{n}\left(Y^{n}\right) \text { is }\left\{\begin{array}{l}
\text { infinite cyclic if } n=1, \\
\text { the direct sum of } k_{n} \text { infinite, } \\
\text { cyclic groups if } n \geqq 2 .
\end{array}\right.
$$

Theorem 2 states that under the given hypotheses, if $Y^{n}$ has properties $1^{n}$ and $2^{n}$, it also has property $5^{n}$.

Two spaces $Y$ and $Z$ are said to have the same homotopy type provided there exist mappings $\alpha: Y \rightarrow Z$ and $\beta: Z \rightarrow Y$ such that $\alpha \beta$ and $\beta \alpha$ are homotopic to the appropriate identity mappings. We denote the identity mapping of a space by the symbol 1 . 
LEMmA 3. Let $Y$ and $Z$ be absolute neighborhood retracts with the same homotopy type. Then $Y$ is a test space for dimension $n$ if and only if $Z$ is.

Proof. Let $Z$ be a test space for dimension $n$. We first show that $Y$ has property $1^{n}$. Let $X$ be a space satisfying the relation $X \tau Y$. To show $\operatorname{dim} X \leqq n$, let $C$ be a closed subset of $X$ and consider any mapping $f: C \rightarrow Z$. Let $\alpha, \beta$ be as above. Then $\beta f: C \rightarrow Y$. Since we are given $X \tau Y, \beta f$ has an extension $F: X \rightarrow Y$ and this provides a mapping $\alpha F: X \rightarrow Z$. Now $\alpha F \mid C=\alpha \beta f$ and since $\alpha \beta \sim 1$, we have $\alpha F \mid C \sim f$. An application of Borsuk's Theorem [3] now yields an extension of $f$ over $X$. Since $Z$ is a test space for dimension $n$, this shows that $\operatorname{dim} X \leqq n$.

An argument similar to that above shows that $Y$ also has property $2^{n}$, which completes the proof of the theorem.

REMARK. The above theorem can be strengthened slightly. It is not necessary that $Y$ and $Z$ have the same homotopy type. All that is needed are mappings $\alpha_{i}: Y \rightarrow Z$ and $\beta_{i}: Z \rightarrow Y(i=1,2)$ such that $\alpha_{1} \beta_{1} \sim 1$ and $\beta_{2} \alpha_{2} \sim 1$, and it is not necessary that $\alpha_{1}=\alpha_{2}, \beta_{1}=\beta_{2}$. Also, $Y$ need not be an absolute neighborhood retract for the first part of the theorem.

LEMma 4. Let $\Sigma^{1}=S^{1}$ and if $n$ is an integer $\geqq 2$ let $\Sigma^{n}$ be the union of $k_{n} n$-spheres $S_{1}^{n}, \cdots, S_{k_{n}}^{n}\left(k_{n}\right.$ determined from Theorem 2) with a single common point. Then $\Sigma_{n}$ is a test space for dimension $n$.

Proof. $\Sigma^{1}$ is, of course, a test space for dimension 1 . Now suppose $n \geqq 2$. Clearly, $\Sigma^{n}$ is an absolute neighborhood retract, so it is locally connected in all dimensions. Also, note that $\pi_{i}\left(\Sigma^{n}\right)=\pi_{i}\left(S_{1}^{n}\right)+\ldots$ $+\pi_{i}\left(S_{\boldsymbol{k}_{n}}^{n}\right), 2 \leqq i \leqq n\left[4\right.$, p. 92], and since $\pi_{i}\left(S_{j}^{n}\right)=0$ for $i<n, j=1, \cdots$, $k_{n}$, we have $\pi_{i}\left(\Sigma^{n}\right)=0, i<n$. According to Theorem 1 , this shows that $\Sigma^{n}$ has property $2^{n}$.

To show that $\Sigma^{n}$ has property $1^{n}$, let us be given a space $X$ satisfying the relation $X \tau \Sigma^{n}$. Let $C$ be a closed subset of $X$ and consider any mapping $f: C \rightarrow S_{1}^{n} \subset \Sigma^{n}$. We are given an extension $F: X \rightarrow \Sigma^{n}$ of $f$. Now it is easy to see that there exists a retraction $r: \Sigma^{n} \rightarrow S_{1}^{n}$. Therefore $r F$ is an extension of $f$ over $X$ (relative to $S_{1}^{n}$ ) and this proves that $\operatorname{dim} X \leqq n$.

Theorem 3. Let $n$ be an integer $\geqq 1$ and let $Y^{n}$ be a compact, $n$ dimensional $n-L C$ space. If $n=1$, we also assume that $Y^{1}$ is 1-simple. If $Y^{n}$ has properties $3^{n}, 4^{n}$ and $5^{n}$, then $Y^{n}$ also has property $1^{n}$.

Proof. If $n=1$, a theorem of S. T. Hu $\left[5\right.$, p. 358] shows that $Y^{1}$ has the same homotopy type as $S^{1}$, so $Y^{1}$ is a test space for dimension 1 by Lemma 3. 
Now suppose that $n \geqq 2$. By property $5^{n}, \pi_{n}\left(Y^{n}\right) \approx \pi_{n}\left(S_{1}^{n}\right)+\cdots$ $+\pi_{n}\left(S_{k_{n}}^{n}\right)$. Therefore $\pi_{n}\left(Y^{n}\right) \approx \pi_{n}\left(\Sigma^{n}\right)$. Since $Y^{n}$ is compact, LC ${ }^{n}$, and $n$-dimensional, we conclude that $Y^{n}$ is an absolute neighborhood retract. Applying a theorem of S. T. Hu [5, p. 357], we see that $Y^{n}$ and $\Sigma^{n}$ have the same homotopy type. Lemmas 3 and 4 now show that $Y^{n}$ is a test space for dimension $n$, completing the proof.

Theorems 1, 2 and 3 give us the main result of this paper:

THEOREM 4. Let $n$ be an integer $\geqq 1$ and let $Y^{n}$ be a compact, $n$ dimensional $n$-LC space. If $n=1$, we also assume that $Y^{1}$ is 1-simple. Then $Y^{n}$ is a test space for dimension $n$ if and only if: (i) $\pi_{i}\left(Y^{n}\right)=0$, $i=0,1, \cdots, n-1$. (ii) $Y^{n}$ is $L C^{n-1}$. (iii) $\pi_{1}\left(Y^{1}\right)$ is infinite cyclic if $n=1 ; \pi_{n}\left(Y^{n}\right)$ is the direct sum of $k_{n}$ infinite cyclic groups if $n \geqq 2$.

We close by briefly mentioning test spaces for dimension 0 . By our previous remark, if $\operatorname{dim} X=0$, then $X \tau Y$ for any space $Y$. On the other hand, it is easy to see that if $Y$ is not connected, then a 0 -sphere (consisting of the union of two points) is a retract of $Y$ and hence $X \tau Y$ implies $\operatorname{dim} X=0$. Thus any space which is not connected is a test space for dimension 0 . This condition is, of course, not necessary.

\section{BIBLIOGRAPHY}

1. C. Kuratowski, Topologie, vol. II.

2. W. Hurewicz, Beiträge zur Topologie der Deformationen III and IV, Proc. Akad. Amsterdam vol. 39 (1936).

3. W. Hurewicz and H. Wallman, Dimension theory, Princeton, 1948, p. 86.

4. P. J. Hilton, An introduction to homotopy theory, Cambridge University Press, 1953.

5. S. T. Hu, Mappings of a normal space into an absolute neighborhood retract, Trans. Amer. Math. Soc. vol. 64 (1948).

6. K. Borsuk, Sur les prolongements des transformations continues, Fund. Math. vol. 28 (1939) p. 108.

RUTGERS UNIVERSITY 